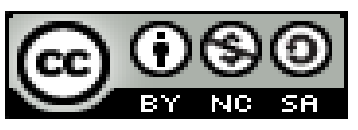

Ciencias de la computación

Artículo de investigación

\title{
Disponibilidad léxica y anglicismos utilizados en informática
}

\section{Lexical and anglicized availability used in computer science}

\section{Disponibilidade léxica e anglicizada usada em ciência da computação}

\author{
Mayra A. Tejena-Macías ${ }^{1}$ \\ mayraatejena@gail.com
}

Recibido: 10 de febrero de 2017 * Corregido: 24 de marzo de $2017 *$ Aceptado: 17 de julio de 2017

\footnotetext{
${ }^{1}$ Magister en Tecnología e Innovación Educativa, Ingeniera en Sistemas, Docente de la Universidad Laica Eloy Alfaro de Manabí, Manta, Ecuador.
} 


\title{
Resumen
}

Este trabajo, evidencia los resultados de un análisis sobre la disponibilidad léxica en torno a tres centros de interés del ámbito de la informática: Internet; software y hardware; y dispositivos de almacenamiento y mediante los programas Dispolex y PASW 17.0. Los resultados muestran que el centro de interés software es en el que más términos ofrecen los participantes; la variable años de estudio de informática tiene incidencia en información adquirida y uso de anglicismos.

Palabras clave: disponibilidad léxica; anglicismos; informática; terminología; software.

\begin{abstract}
This work, shows the results of an analysis on the lexical availability around three centers of interest in the field of information technology: Internet; software and hardware; and storage devices and through the Dispolex and PASW 17.0 programs. The results show that the center of software interest is where the participants offer the most terms; The variable years of computer science study has an impact on acquired information and the use of anglicisms.
\end{abstract}

Keywords: lexical availability; anglicisms; computing; terminology; software.

\section{Resumo}

Este trabalho mostra os resultados de uma análise sobre a disponibilidade lexical em torno de três centros de interesse no campo da tecnologia da informação: Internet; software e hardware; e dispositivos de armazenamento e através dos programas Dispolex e PASW 17.0. Os resultados mostram que o centro de interesse de software é onde os participantes oferecem mais termos; A variável anos de estudo da ciência da computação tem impacto sobre a informação adquirida e o uso de anglicismos.

Palavras chave: disponibilidade lexical; anglicisms; ciência da computação; terminologia; software. 


\section{Introducción}

Existe una estrecha relación entre el inglés y las tecnologías de la información y las comunicaciones (TIC) y, en particular, el de la informática, ya que gran parte de esta es desarrollada en países con supremacía económica como los Estados Unidos, llegándonos así, los avances tecnológicos y el lenguaje que se desarrolla en torno a la informática. Al mismo ritmo al que se han importado de EE UU todos estos productos informáticos, el español, al igual que otros muchos idiomas, también ha ido tomando prestados los términos que denominan estas creaciones. La mayor parte de la terminología informática proviene de calcos del inglés (Belda Medina 2003; Vitoria López 2005). Duque Pérez (1996: 4-5), señala que, debido a su constante desarrollo, su terminología carece de estabilidad léxicosemántica y esto influye en el grado y en la calidad de la comunicación. Es por esta razón que muchos de estos términos pasan a formar parte del vocabulario de quienes se desenvuelven en este campo, sean especialistas o no, ya que no sólo están presentes en publicaciones especializadas, sino que además aparecen en cualquier revista, periódico e incluso folletos comerciales de cualquier establecimiento. Sin embargo, también es cierto que otros muchos de estos vocablos disponen de un período limitado de vida en el uso, pues puede ocurrir que el objeto al que designan quede pronto obsoleto y, por ello, fuera de uso. Todo ello implica que se trata de un campo muy inestable que se desarrolla a un ritmo vertiginoso, y del mismo modo lo hace su terminología. En este contexto, recogemos los resultados de un estudio empírico sobre la disponibilidad léxica de los anglicismos informáticos crudos, realizado en la Universidad de Las Palmas de Gran Canaria entre alumnos de la facultad de Traducción e Interpretación. Los informantes se encuentran en su último año de carrera. En algunas de sus asignaturas el empleo del ordenador es esencial; por tanto, a pesar de tratarse de alumnado del área humanística, cuenta con cierta familiaridad con el argot informático. La selección de estudiantes de traducción no responde al azar. En un campo cuyos principales adelantos siguen siendo importados de Estados Unidos, los traductores adquieren una gran responsabilidad como difusores de la terminología informática y han de ser capaces de adoptar un criterio coherente entre posiciones "puristas", que abogan por una norma "fantasma"18, teóricamente capaz de evitar la entrada excesiva de anglicismos innecesarios en nuestro idioma, y los que defienden el criterio de uso y la inevitable evolución lingüística como única brújula (Bolaños Medina, 2003). De ahí el interés de 
estudiar los hábitos de uso de los anglicismos crudos informáticos en este sector profesional. Con el presente trabajo pretendemos ofrecer datos actualizados acerca de la disponibilidad léxica y la presencia de anglicismos en el español en el campo de la informática.

\section{Material y métodos}

La finalidad de este trabajo es obtener información sobre la disponibilidad léxica de los anglicismos crudos que forman parte de la terminología informática disponible. Se trata de un análisis descriptivo de los resultados obtenidos tras la aplicación de una encuesta realizada a los estudiantes de la carrera de informática en Manta. La ejecución de la prueba fue supervisada con el fin de evitar que los estudiantes consultaran fuentes documentales en los equipos de consulta disponibles y que intercambiaran información entre ellos. El objetivo fue obtener información sobre una serie de variables de interés, tales como si habían recibido formación específica previa sobre informática, así como sus años de estudio de lengua inglesa y en la facultad, entre otros.

La única excepción realizada se refiere al tratamiento de las marcas comerciales. Muchos de los términos recopilados en este trabajo aluden a marcas o nombres propios de programas informáticos, sitios web, redes sociales, etc. Resulta prácticamente inevitable su aparición, ya que se trata de vocabulario que designa a aplicaciones informáticas que en su mayoría provienen de Estados Unidos, por tanto, normalmente está presente el nombre del fabricante (por ejemplo, Microsoft, Apple) y el nombre del producto informático (PowerPoint, Excel, iPod, etc.). En este caso, a pesar de que las citadas directrices aconsejan la supresión de las que no estén lexicalizadas, se optó por mantenerlas en su totalidad, atendiendo a dos criterios principales: su gran frecuencia de aparición entre los datos recopilados, en particular, en el ámbito del software y el carácter difuso de los límites que demarcan lo que está lexicalizado de lo que no lo está, en particular cuando estudiamos el lenguaje especializado de la informática. Otras dificultades encontradas han sido la eliminación de términos repetidos, y, en mayor medida, la corrección de la ortografía. Muchas de las palabras presentes en este estudio son anglicismos no aceptados por el diccionario de la RAE y tampoco aparecen en el Diccionario Panhispánico de dudas, y suelen presentar una ortografía problemática, cuando menos, inconsistente. 
Esto hizo que se tuviera que adoptar un criterio ortográfico para ellas y mantenerlo, para conseguir cierta coherencia.

\section{Marco teórico}

\section{La disponibilidad léxica}

La creación del Proyecto panhispánico de estudio coordinado del léxico disponible bajo la dirección de Humberto López Morales (1996) en los años noventa constituye sin duda su principal hito. Desde entonces, la disponibilidad léxica no ha cesado de atraer el interés de los investigadores (López Morales y García Marco 1995; López Morales 1999; Bartol Hernández 2003), posiblemente debido a sus aplicaciones en los más diversos ámbitos (Samper Padilla y Samper Hernández 2006); desde la lingüística aplicada a la enseñanza de la lengua (Carcedo González 1998; Samper Henández 2003; Sánchez Gómez 2005; Samper Hernández 2014), hasta la psicolingüística (Hernández Muñoz 2006), sin olvidar la dialectología (López Chávez 1992; Samper Padilla 1999; Samper Padilla, Hernández Cabrera y Bellón Fernández 2003; Samper Hernández 2005) e incluso la etnolingüística (Carcedo González 1998). Asimismo, los estudios de disponibilidad léxica en el español han dado lugar a un gran número de trabajos y cuatro encuentros. En su discurso inaugural del IV Encuentro de disponibilidad léxica, "La Universidad del Español”, celebrado en Salamanca en abril de 2011, Borrego Nieto, su coordinador, resume con gran acierto los logros alcanzados hasta el momento en este campo. Este autor señala que "las palabras muestran una configuración asombrosamente parecida en el mundo hispánico, pese a la inmensidad de sus tierras, no obstante, aparecen aquí localismos y dialectismos que merece la pena estudiar porque son significativos". A este respecto, convendría también añadir los extranjerismos, y en particular anglicismos, que se introducen en el español y que, sin duda, requieren de mayor atención. El concepto de disponibilidad léxica se refiere al "caudal léxico utilizable en una situación comunicativa dada, solo que, si esta no se producía, ciertas unidades léxicas no se realizaban” (López Morales, 1996: 246). Por consiguiente, el índice de disponibilidad léxica se calcula teniendo en cuenta la frecuencia de aparición de cada vocablo en la lista proporcionada por cada sujeto de la muestra de estudio con las palabras que acuden de forma instantánea a su mente ante 
un estímulo temático, atendiendo también a la posición en que aparece cada una de ellas y al número de informantes. Los experimentos de disponibilidad lingüística son pruebas de fluencia semántica o de categoría, tareas asociativas bajo supervisión que cuentan con una larga tradición en el ámbito de la psicología experimental (Clark 1970; Damasio 1990; Caramazza 1998). En su búsqueda de relaciones entre el pensamiento conceptual, la experiencia corpórea y la estructura lingüística, la lingüística cognitiva recurre al concepto de categorización como mecanismo de apropiación de la realidad, y establece que "las categorías semánticas lingüísticas no sean autónomas respecto a la organización de las categorías conceptuales generales" (Hernández-Muñoz, 2006: 38). Esta categorización cobra sentido en el uso de los centros de interés o categorías en los experimentos sobre léxico disponible. Tal como señala Borrego Nieto (2011), los centros de interés o categorías investigados en los estudios de disponibilidad no son homogéneos; algunos funcionan como clases naturales, otros como campos asociativos, algunos son más compactos, mientras que otros son más difusos. Algunos campos generan mayor cantidad de vocabulario que otros, y también los hay que son más sensibles que otros a ciertas variables. Este mismo autor ha señalado que variables como el género y el ámbito rural o urbano son cambiantes y tienen un influjo relativamente poco perceptible sobre la cantidad de léxico disponible, frente a otras, como el estatus social y la cualidad de la educación, que aparecen como un factor clave en la competencia léxica de los informantes (Samper Hernández, 2009). También se ha descrito un proceso de debilitamiento progresivo de la memoria a largo plazo con la edad que podría afectar al rendimiento en este tipo de pruebas (Urrutia, 2001). En este tipo de experimentos se ha comprobado la existencia de dos fenómenos que podrían facilitar la tarea: la creación de agrupaciones o subgrupos, fácilmente detectables dentro de los centros de interés o categorías generales que ayudan a organizar la producción de los vocablos, y los cambios de subgrupo; y la habilidad de un informante para pasar de un tema a otro dentro de la misma categoría (Troyer, Moscovitch y Wincour, 1997). Asimismo, se ha comprobado que "cuanto mayor sea la habilidad para crear grupos y cambiar entre ellos, mayor número de palabras serán las evocadas" (Hernández-Muñoz, 2006:105) y que también existen procesos inconscientes, como el priming19, que pueden influir en los resultados (Troyer, Moscovitch y Wincour, 1997). Cabe destacar que este tipo de experimentos entrañan ciertas dificultades metodológicas; tres son los problemas principales

725 Vol. 3, núm. 4, octubre, 2017, pp. 720-738 Mayra A. Tejena-Macías 
que escapan al control del investigador según Hernández-Muñoz (2006): como hemos visto, los informantes pueden utilizar estrategias que les permitan realizar la tarea de una forma más simple y con menor esfuerzo; un mismo producto o resultado puede haber sido motivado por distintas causas que no podemos identificar; el experimento en sí es una "actividad artificial", que no tiene por qué reproducir el mismo proceso tal y como sucede en el contexto comunicativo habitual. Sin embargo, a pesar de estos inconvenientes metodológicos, aún con sus limitaciones, consideramos que se trata de un método adecuado para obtener información sobre el lexicón con fines precisos (op. cit.). Por último, se han delimitado estadísticamente tres variables predictoras de la disponibilidad léxica, y son, según su grado de influencia: la tipicidad, la edad de adquisición y la familiaridad del concepto (HernándezMuñoz, Izura y Ellis, 2006). Sin embargo, se ha encontrado que otras variables estudiadas, como la longitud o la imaginabilidad, no desempeñan un papel de interés.

\section{Los anglicismos en español}

Existen muchas definiciones para el término anglicismo. Sin embargo, partiremos de la que aporta el diccionario de la Real Academia Española con sus tres acepciones: 1. Giro o modo de hablar propio de la lengua inglesa; 2. Vocablo o giro de esta lengua empleado en otra; 3. Empleo de vocablos o giros ingleses en distintos idiomas. Tal como señala Rodríguez Segura (1999: 17), ciertas causas favorecen estos usos: la necesidad de recurrir a un término inglés que no se conoce porque el empleo del anglicismo evita locuciones engorrosas en español; porque el anglicismo es un término o palabra más general o más impreciso para referirse a algo; por eufemismo; por cubrir un hueco surgido cuando otras palabras han desplazado su significado y han dejado un vacío semántico para el nuevo concepto u objeto; para dar prestigio a un determinado producto; como indicador de especialización en algún tema; para lograr un efecto concreto en el oyente o lector (cómico, inesperado, etc.); por la necesidad de lexicalizar nuevos inventos y descubrimientos técnicos o científicos y tener que recurrir a un término inglés ya que no existe un equivalente en español; y por esnobismo o afán de mostrar cierto nivel intelectual. La masiva presencia de anglicismos léxicos se evidencia, en nuestra lengua, en múltiples campos, como el de la economía y las finanzas (Sevilla Muñoz y Sevilla Muñoz 1996; Gómez Moreno 1996), el del dominio legal (Sánchez y Durán 2002), la publicidad (Durán 2002;

726 Vol. 3, núm. 4, octubre, 2017, pp. 720-738

Mayra A. Tejena-Macías 
Gerritsen et al. 2007; Rodríguez-Díaz 2011), la publicidad infantil (Luján-García 2011), la música (Olivares 2009), los escaparates (Luján-García 2010) y, por supuesto, la informática (Ramírez Verdugo 1995; Pérez y Vivanco 1997; Sampedro Losada 2000; Jiménez y Mandado 2008; Cerdá et al. 2005; Solís 2005; Márquez y Lorente 2006; Pano 2007; Berglund 2008; Bolaños y Luján 2010), que es sumamente productivo y abierto a este tipo de préstamos, como demuestra este gran número de trabajos. En el ámbito aplicado, debemos también destacar la presencia de diferentes estudios (Paredes García 2000; González y Orellana 2006) que han analizado la disponibilidad de anglicismos léxicos entre estudiantes preuniversitarios. Estos trabajos tienen el denominador común de haber empleado el cuestionario como instrumento de análisis para obtener resultados que reflejan la creciente popularidad e inserción de anglicismos en el español, tanto para designar conceptos que ya cuentan con su término en español, como para llenar un vacío léxico en nuestra lengua. 2.3. Los anglicismos en la informática El empleo de anglicismos en la informática es un campo de investigación muy complejo, debido a su inestabilidad y vertiginoso ritmo de aparición, dada la evolución tan rápida en los avances tecnológicos. En esta sección, haremos referencia a algunos estudios destacados realizados en este terreno, ya que sería imposible abarcar en un único artículo la totalidad de los trabajos que tratan esta cuestión. Convendría señalar que aunque la mayoría de las investigaciones que tratan este tema se han centrado en el nivel léxico-semántico, dado que es en éste donde la influencia del inglés es más obvia, también se aprecia su huella de manera notable en el nivel sintáctico. Así lo demuestran estudios como los realizados por Rodríguez Segura (1999) y Rodríguez Medina (2000), quienes analizan de manera rigurosa los distintos calcos importados del inglés al español. Esta última autora examina los casos de interferencias (los calcos que se producen en la sintaxis española como resultado de la transferencia de estructuras sintácticas inglesas inexistentes en español) en manuales informáticos y encuentra usos de preposiciones, sustantivos, verbos y adjetivos en español que responden a una clara influencia de la lengua inglesa.

Al referirnos a los nombres propios, Márquez y Lorente (2006) se han ocupado de desvelar la ausencia de traducción en la mayoría de los de esta área. De este modo, vocablos como Apple, Pentium, PowerPoint, Microsoft Windows, Facebook, You Tube, eBay, por mencionar sólo unos pocos, y muy frecuentes en nuestra investigación, como veremos, pasan a formar parte de nuestro vocabulario

727 Vol. 3, núm. 4, octubre, 2017, pp. 720-738

Mayra A. Tejena-Macías 
habitual sin adaptación morfológica ni ortográfica y sin que medie ningún término equivalente en español. Estos autores han observado también cómo un nombre propio "puede dejar de serlo en ciertos contextos, por lexicalización pragmática, semántica e incluso formal" (op. cit., 1). Recientemente han visto la luz varios glosarios y diccionarios en línea especializados en informática en formato digital, que presentan la inestimable ventaja de ser, en potencia, mucho más fácilmente actualizables. Entre ellos, destaca el Vocabulario de ordenadores e Internet de José Antonio Millán (1998), que ofrece, además de propuestas de traducción, información etimológica y lingüística de interés, a menudo acompañada de ejemplos. Otra obra digna de mención es el Glosario básico inglés-español para usuarios de Internet (Fernández Calvo 1994), que incluye también las definiciones de los términos. Asimismo, coincidimos con Vitoria López (2005) en destacar el importante papel que están teniendo tanto los grupos de debate como las listas de distribución (tales como Tecnotrad y Tradumática, ofrecidas por el Servicio de Listas de Distribución de RedIris) para facilitar el debate sobre aspectos terminológicos. No son sólo los lingüistas y académicos los que parecen adoptar diversas posturas con respecto a esta cuestión, también lo hacen algunos ingenieros e informáticos. Por un lado, encontramos una actitud purista que muestra su rechazo ante el uso indiscriminado y masivo de términos en inglés (Vaquero 1999; López Jiménez 1999). En este sentido, Jiménez y Mandado (2008) aportan todo un listado de términos en español que podrían sustituir a otros tantos vocablos en inglés. Algunos ejemplos podrían ser "ruta de datos" en lugar de datapath, "pasarela" en lugar de gateway o "memoria intermedia" o "circuito de salida" en vez de buffer. Estos autores aluden al uso de estos préstamos con cierta intencionalidad esnobista. No obstante, como afirma Vitoria López (2005: 114), muchas veces se prefiere el anglicismo al término traducido por dos razones principales: porque técnicos e informáticos desconocen las reglas de formación de palabras en español y no muestran interés por investigar sobre el significado del término extranjero; y por la mayor brevedad que suele caracterizar a los términos ingleses frente a los de nuestro idioma, lo que revierte en una mayor comodidad de uso. Por otro lado, existen también otros argumentos basados en el concepto de homogeneidad, es decir, el empleo de términos técnicos afines a cualquier lengua, la búsqueda de una terminología internacional que facilite tanto la comunicación entre profesionales como la expansión comercial de los productos informáticos (Belda Medina, 2003). De este modo, por ejemplo, tanto en 
alemán como en inglés, en italiano o en español se va a emplear un mismo término para referirse al mismo concepto. Topamos aquí con la globalización y la tendencia a la uniformidad de los lenguajes especializados. Por último, también está la postura que se limita a describir este fenómeno de forma documentada y neutral, sin ofrecer resistencia ni protección de estos términos en español. En esta línea se inscribe el presente trabajo.

La rapidez con la que se mueven las Tecnologías de la Información y las Comunicaciones hace que estos términos técnicos no cuenten con un criterio bien determinado al introducirse en el español. En este sentido, Lázaro Carreter (en Pano, 2007: 6) señala que la avalancha de nuevos términos técnicos que denominan las numerosas invenciones y descubrimientos es tan grande que se con frecuencia se actúa "con prisas", sin "criterio fijo" y de acuerdo con las siguientes posibilidades: adoptar pura y simplemente el tecnicismo extranjero; adaptarlo de manera apresurada, con leves e imprescindibles retoques fónicos; o sustituir el extranjerismo por una palabra o un giro del idioma propio. Con el objeto de ver el grado de integración y naturalización de estos términos en el español, emplearemos la siguiente clasificación propuesta por Lorenzo (1996): anglicismos crudos, anglicismos adaptados, calcos anglicados y anglicismos híbridos. Consideramos anglicismos crudos aquellos que mantienen en español la grafía inglesa y un reflejo de la pronunciación originaria (batch, firewall, hacker, etc.); son anglicismos adaptados aquellos que han adaptado su morfología de forma total o parcial al español. En el caso de estos vocablos resulta más difícil reconocer el origen anglosajón del término, ya que se han incorporado plenamente en la lengua española desde hace cierto tiempo. Algunos ejemplos son computadora, escáner, impresora, etc.; son calcos anglicados aquellos préstamos provenientes del inglés que imitan el significado o estructura de una palabra o expresión inglesa y no su entidad fonética. Por ejemplo, disco duro por el inglés hard disk. Los calcos semánticos son voces españolas que, por su semejanza formal con otras inglesas, reciben de este idioma acepciones que no poseían en español: mouse (ratón), tools (herramientas), etc. Los anglicismos híbridos son aquellos vocablos cuyos morfemas provienen de dos lenguas distintas, una de ellas el inglés. Algunos ejemplos son puenting o balconing, cuyos lexemas proceden del español más el morfema -ing. Se trata, además, de términos que no existen en inglés con esta ortografía. No hay que olvidar que algunos indicativos sobre el proceso de integración o aclimatación de estos términos son, tal como indica Berns (1994:

\section{9} Vol. 3, núm. 4, octubre, 2017, pp. 720-738 Mayra A. Tejena-Macías 
182-183), convenciones de carácter formal tal como el uso de guiones dentro de los términos. Así, por ejemplo, en nuestro estudio observamos que el vocablo e-mail emplea guiones, lo cual nos hace pensar que se trata de palabras que se encuentran en proceso de integración, a pesar de la existencia de equivalentes alternativos reconocidos por la RAE como "correo electrónico". En cuanto al término on line, éste ha sido reconocido por el diccionario Panhispánico de dudas, aunque no por el de la RAE. La ortografía on line, sin guión, es la propuesta por el glosario ATI (Asociación de Técnicos de informática) de Fernández Calvo (1994). Vemos, pues, que a pesar de las fuentes ya arriba mencionadas, se trata este de un ámbito que exige una actualización y revisión constantes y del que se ha señalado "la escasez de estudios sistemáticos y rigurosos sobre el tema" (Cerdá et al., 2005: 247).

\section{Resultados}

A continuación, se exponen los resultados más relevantes obtenidos del análisis de los datos recopilados de estudiantes de Traducción. En los casos en los que puede resultar ilustrativo para los fines de la investigación, se establece la comparación de algunos de los datos descritos en los apartados siguientes con los hallados en una segunda encuesta paralela preliminar realizada a estudiantes de informática.

\section{Índices cuantitativos}

Los estudiantes de traducción produjeron una media de 632,33 palabras y 255 vocablos 21 por centro de interés, lo que representa una media de 15,81 palabras y 6,37 vocablos por informante. En la Tabla 1 se resumen los índices cuantitativos por centro de interés. Se aprecia que el centro de interés que suscitó el mayor número de respuestas fue Internet, seguido del software y, por último, delhardware.

730 Vol. 3, núm. 4, octubre, 2017, pp. 720-738

Mayra A. Tejena-Macías 
Disponibilidad léxica y anglicismos utilizados en informática

\begin{tabular}{|l|l|l|l|l|}
\hline & $\begin{array}{l}\text { Palabras } \\
\text { totales }\end{array}$ & Vocablos & $\begin{array}{l}\text { Palabras por } \\
\text { informante }\end{array}$ & $\begin{array}{l}\text { Vocablos por } \\
\text { informante }\end{array}$ \\
\hline Internet & 696 & 291 & 17,4 & 7,2 \\
\hline Hardware & 621 & 216 & 15,5 & 5,4 \\
\hline Software & 580 & 258 & 14,5 & 6,4 \\
\hline
\end{tabular}

Tabla 1. Índices generales de estudiantes de Traducción

Si comparamos estos datos con los recabados de los estudiantes de informática, comprobaremos que el orden de los centros de interés según el número de vocablos producidos por los informantes varía: en primer lugar, se sitúan el software y el hardware, y, en último lugar, Internet. La media de palabras totales se sitúa en 125,33 y la de vocablos en 83, es decir, una media de 13,92 palabras y 9,21 vocablos por informante. Llama la atención que, aunque el número de palabras recopiladas ha sido un poco más elevado entre los estudiantes de traducción, son los de informática los que aportan una media de vocablos más alta. Esto puede quizá deberse al mayor dominio de la terminología específica por parte de estos últimos y a la necesidad de los primeros de recurrir, en ocasiones, a términos más explicativos que técnicos, como por ejemplo, "programas relacionados con la traducción" o "sistema gestor de base de datos terminológicos", en el caso de los estudiantes de traducción. En la tabla 2 aparecen, de forma resumida, los índices cuantitativos por centros de interés de los estudiantes de informática.

\begin{tabular}{|l|l|l|l|l|}
\hline & $\begin{array}{l}\text { Palabras } \\
\text { totales }\end{array}$ & Vocablos & $\begin{array}{l}\text { Palabras por } \\
\text { informante }\end{array}$ & $\begin{array}{l}\text { Vocablos por } \\
\text { informante }\end{array}$ \\
\hline Internet & 117 & 76 & 13 & 8,4 \\
\hline Hardware & 144 & 86 & 16 & 9,5 \\
\hline Software & 115 & 87 & 12,7 & 9,6 \\
\hline
\end{tabular}

Tabla 2. Índices generales de estudiantes de Informática. 


\section{Incidencia de la variable género}

En la Tabla 3 se recogen los índices cuantitativos de los informantes de género masculino, con una media de 141 palabras y 85 vocablos por centro de interés, frente a la media de 468 palabras y de 211,3 vocablos de los informantes de género femenino (ver Tabla 4).

\begin{tabular}{|l|l|l|l|l|}
\hline $\mathrm{N}=8$ & $\begin{array}{l}\text { Palabras } \\
\text { totales }\end{array}$ & Vocablos & $\begin{array}{l}\text { Palabras por } \\
\text { informante }\end{array}$ & $\begin{array}{l}\text { Vocablos } \\
\text { por infor- } \\
\text { mante }\end{array}$ \\
\hline Internet & 136 & 92 & 17 & 11,5 \\
\hline $\begin{array}{l}\text { Hardware y } \\
\text { dispositivos de } \\
\text { almacenamiento }\end{array}$ & 151 & 79 & 18,8 & 9,8 \\
\hline Software & 136 & 84 & 17 & 10,5 \\
\hline
\end{tabular}

Tabla 3. Índices cuantitativos de los informantes de género masculino

Sin embargo, tanto el número medio de palabras por informante como el de vocablos por informante es ligeramente mayor en el caso de los estudiantes de género masculino, lo que se aprecia si contrastamos las 17,6 palabras y 10,6 vocablos de media entre éstos últimos con las 15,09 palabras y los 6,8 vocablos de los estudiantes de género femenino.

\begin{tabular}{|l|l|l|l|l|}
\hline $\mathrm{N}=31$ & $\begin{array}{l}\text { Palabras } \\
\text { totales }\end{array}$ & Vocablos & $\begin{array}{l}\text { Palabras por } \\
\text { informante }\end{array}$ & $\begin{array}{l}\text { Vocablos } \\
\text { por infor- } \\
\text { mante }\end{array}$ \\
\hline Internet & 536 & 253 & 17,2 & 8,1 \\
\hline $\begin{array}{l}\text { Hardware y } \\
\text { dispositivos de } \\
\text { almacenamiento }\end{array}$ & 443 & 175 & 14,2 & 5,6 \\
\hline Software & 425 & 206 & 13,7 & 6,6 \\
\hline
\end{tabular}

Tabla 4. Índices cuantitativos de los informantes de género femenino 


\section{Anglicismos y años de formación en informática}

El mayor número de anglicismos se ha registrado entre los estudiantes que han recibido una formación en informática más prolongada y, el menor, entre aquellos que la han estudiado entre 0 y 5 años, situándose en un término medio los que lo han hecho durante un periodo de entre 6 y 10 años. De nuevo, las cifras más elevadas se han obtenido en el centro de interés del software y llama la atención que la totalidad de los términos utilizados por el grupo de mayor formación en esta categoría fueron anglicismos.

\begin{tabular}{|l|l|l|l|l|}
\hline & Internet & $\begin{array}{l}\text { Hardware y dispositivos de } \\
\text { almacenamiento }\end{array}$ & Software & Media \\
\hline $0-5$ & $42 \%$ & $38 \%$ & $59 \%$ & $46,3 \%$ \\
\hline $6-10$ & $64,2 \%$ & $36 \%$ & $71,4 \%$ & $57,2 \%$ \\
\hline $11-15$ & $64,7 \%$ & $35,2 \%$ & $100 \%$ & $66,6 \%$ \\
\hline
\end{tabular}

\section{Anglicismos con mayor índice de disponibilidad}

A continuación, analizamos los anglicismos con mayor índice de disponibilidad por centro de interés. En las diferentes tablas se incluirán en orden decreciente y acompañados también de su frecuencia relativa. Es necesario aclarar que hemos incluido en este grupo aquellos términos compuestos por más de un vocablo y que podrían considerarse como híbridos; es decir, aquellos en los que uno de los dos elementos es una forma castellana y el otro un término proveniente del inglés sin adaptación morfológica ni ortográfica.

\section{Anglicismos con mayor índice de disponibilidad en el ámbito de Internet}

En la tabla 14 aparece la lista de los anglicismos con mayor índice disponibilidad en este ámbito. Es necesario subrayar los siguientes hechos: entre las formas híbridas, las compuestas con el vocablo web (página web y sitio web), se sitúan entre las 6 primeras; también destaca el caso de on line, aunque cuenta con un menor índice de disponibilidad ("diccionario on line" y "traductor on line"). 
Disponibilidad léxica y anglicismos utilizados en informática

\begin{tabular}{l|l|l|l|l|l|}
\hline Palabra & Disponib. & Frec. Relativa & Palabra & Disponib. & Frec. relativa \\
\hline página web & 0,56059 & $3,655 \%$ & Mozilla & 0,06506 & $0,585 \%$ \\
\hline Google & 0,27132 & $2,339 \%$ & Skype & 0,05188 & $0,439 \%$ \\
\hline chat & 0,25482 & $2,485 \%$ & Word & 0,04685 & $0,439 \%$ \\
\hline blog & 0,24399 & $2,193 \%$ & Wikipedia & 0,04555 & $0,585 \%$ \\
\hline web & 0,19715 & $1,170 \%$ & download & 0,04300 & $0,292 \%$ \\
\hline sitio web & 0,14438 & $0,877 \%$ & eBay & 0,04126 & $0,439 \%$ \\
\hline wifi & 0,13958 & $1,462 \%$ & Explorer & 0,03946 & $0,439 \%$ \\
\hline e-mail & 0,13699 & $1,316 \%$ & $\begin{array}{l}\text { Wordref- } \\
\text { erence }\end{array}$ & 0,03599 & $0,292 \%$ \\
\hline Facebook & 0,13535 & $1,170 \%$ & Multiterm & 0,03388 & $0,292 \%$ \\
\hline link & 0,12369 & $0,877 \%$ & Windows & 0,03362 & $0,292 \%$ \\
\hline spam & 0,10795 & $0,877 \%$ & HTML & 0,03273 & $0,292 \%$ \\
\hline ADSL & 0,10613 & $1,023 \%$ & $\begin{array}{l}\text { Internet } \\
\text { Explorer }\end{array}$ & 0,03034 & $0,292 \%$ \\
\hline $\begin{array}{l}\text { diccionario } \\
\text { on line }\end{array}$ & 0,09956 & $0,877 \%$ & click & 0,02877 & $0,292 \%$ \\
\hline Messenger & 0,09240 & $0,877 \%$ & $\begin{array}{l}\text { Power- } \\
\text { point }\end{array}$ & 0,02836 & $0,292 \%$ \\
\hline software & 0,08798 & $0,585 \%$ & USB & 0,02500 & $0,146 \%$ \\
\hline on line & 0,08657 & $0,877 \%$ & eMule & 0,02428 & $0,292 \%$ \\
\hline
\end{tabular}

\begin{tabular}{l|l|l|l|l|l|} 
You Tube & 0,07999 & $1,023 \%$ & FAQ & 0,02353 & $0,146 \%$ \\
\hline URL & 0,07886 & $0,585 \%$ & $\begin{array}{l}\text { traductor } \\
\text { on line }\end{array}$ & 0,02353 & $0,146 \%$ \\
\hline Tuenti & 0,07863 & $0,731 \%$ & www & 0,02353 & $0,146 \%$ \\
\hline router & 0,07430 & $0,731 \%$ & Twitter & 0,02343 & $0,292 \%$ \\
\hline cookies & 0,07086 & $0,585 \%$ & Access & 0,02289 & $0,292 \%$ \\
\hline Hotmail & 0,06883 & $0,731 \%$ & hardware & 0,02215 & $0,146 \%$ \\
\hline
\end{tabular}

El 45,45\% de los anglicismos crudos identificados son nombres propios; de ellos, un 55\% está constituido por programas informáticos propiamente dichos; el resto está relacionado directamente con Internet. En el caso de Tuenti, la competencia de Facebook de origen español, el nombre surgió del deseo de los creadores de combinar "tú” y “ti”" (Simón, 2008), y eligieron el que sería el definitivo por hacer alusión en inglés a las edades de los usuarios a los que iba destinado (en su mayoría veinteañeros) y por recordar también a "tu entidad". Dado que se trata de un juego de palabras que fonológicamente constituye un anglicismo crudo, y sobre todo, debido al interés que despierta su elevada frecuencia de aparición, hemos decidido tratarlo como tal. El 13,6 del total de anglicismos de este centro de interés son siglas, aunque ninguna de ellas aparece entre los 10 términos más disponibles. Destaca asimismo la elevada disponibilidad de vocablos relacionados directamente con la traducción, por ejemplo, "Multiterm" (un software de gestión terminológica integrado con un sistema de memoria de traducción), o "diccionario on line" y "traductor on line". 


\section{Conclusiones}

Iniciamos esta sección de conclusiones haciendo hincapié en su carácter preliminar, al tratarse de un estudio que en un futuro se verá complementado por una muestra más amplia y variada, con alumnado de varias titulaciones, con el fin de establecer comparativas fiables. De cualquier modo, coincidimos con Hernández-Muñoz (2006) en señalar que de los experimentos de disponibilidad no se puede inferir de forma fiable la realidad del funcionamiento lingüístico, debido, entre otros factores, a las dificultades metodológicas ya comentadas en el marco teórico, pero sí permiten obtener información sobre el lexicón con fines precisos. En cualquier caso, una de las aplicaciones de este estudio y de otros afines podría ser su uso con fines didácticos a la hora de sensibilizar a los estudiantes de traducción informática sobre la realidad del uso de esta terminología, su responsabilidad como principales vehículos de transmisión de la tecnología propia los avances tecnológicos del inglés al español y de su denominación, así como de la conveniencia de establecer unos parámetros que permitan la utilización de otros recursos neológicos más propios de nuestro idioma allí donde todavía sea posible. Dada la falta de normalización que caracteriza a la terminología informática, incluso a aquellos vocablos cuyo uso está ya extendido entre la población en general, estos resultados, así como los obtenidos con estudios similares, podrían contribuir también a constituir un criterio racional para la selección de los vocablos que tendrían que aparecer en un diccionario (ver también HernándezMuñoz, Izura y Ellis, 2006) y suplir en parte las carencias terminológicas detectadas en este sentido. En líneas generales y a modo de conclusión se podría afirmar que el análisis de disponibilidad léxica de los estudiantes implicados en este trabajo arroja luz sobre distintos aspectos:

1. Software es el centro de interés que ofrece una mayor disponibilidad léxica de anglicismos entre los informantes, seguido de Internet y hardware, tanto entre estudiantes de traducción como de informática.

2. La variable "años de estudio en informática" parece tener cierta influencia directa, tanto en el grado de disponibilidad léxica como en el número de anglicismos en este campo entre los estudiantes encuestados.

735 Vol. 3, núm. 4, octubre, 2017, pp. 720-738

Mayra A. Tejena-Macías 
3. Por el contrario, la variable "años de estudio del inglés" no ofrece resultados que nos hagan pensar que existe una incidencia notable de esta variable en ninguno de los factores analizados.

4. Se confirma la importancia de la familiaridad con el concepto como variable predictora de la disponibilidad léxica, en particular, en centro de interés de software.

\section{Referencias bibliográficas}

Bartol Hernández, J. A. 2003. "Léxico disponible y norma lingüística.” Lengua, variación y contexto: estudios dedicados a Humberto López Morales. Ed. F. Moreno Fernández, J. A Sámper Padilla, M. Vaquero, M. L. Gutiérrez Araus, C. Hdez Alonso y F. Jimeno Menéndez. (coords.). Madrid: Arco Libros. 127-143.

Belda Medina, José R. 2001. "Computer terminology problems in translating abbreviations and acronyms". Proceedings of First International Conference on Specialized Translation.

Eds. J. Chabás, M. Cases y R. Gaser. Barcelona: Arial. 66-71. 2003. El lenguaje de la informática e internet y su traducción. Alicante: Publicaciones de la Universidad de Alicante.

Benítez Pérez, P. 1992. "Listas abiertas y listas cerradas de palabras en disponibilidad léxica". Actas del VIII Congreso Nacional de Lingüística Aplicada. Ed. J. R. Losada Durán y M. Mansilla García. Vigo: Asociación Española de Lingüística Aplicada. 117-127.

Berglund, M. 2008. “¿Mailing por necesidad o por lujo? Un estudio sobre anglicismos en prensa española”. Högsolan Skövde. Institutionen för Kommunication och information Spanska. 18 marzo 2010 < http://www.his.diva-portal.org. Pdf> Berns, M. 1994. "English in German Legal Writings". American Journal of Germanic Linguistics and Literatures 4, 2: 175-198.

Bolaños Medina, A. 2003. "Dificultades y estrategias de la localización de sitios web comerciales del inglés al español”. Internet in linguistics, translation and literary studies. Eds. S. Posteguillo, e. ortells, J.r. Prado, A. Bolaños y A. Alcina. Castellón: Universitat Jaume I. 241-263. 
Bolaños Medina, A. y Luján García, C. 2010. “Análisis de los anglicismos informáticos crudos del léxico disponible de los estudiantes universitarios de traducción”. Lexis, XXXIV, 2. 241-274.

Borrego Nieto, J. y Fernández Juncal, Ma C. 2003. “¿En qué cambia la universidad la disponibilidad léxica de los preuniversitarios?”. Lengua, variación y contexto: estudios dedicados a Humberto López Morales. Eds. F. Moreno Fernández, J. A. Samper Padilla, M. Vaquero, M.1. Gutiérrez Araus, C. Hedz. Alonso Y F. Jimeno Menéndez. (coords). Madrid: Arco Libros. 167-178. Borrego Nieto, J. 2011. Discurso inaugural del IV Encuentro de disponibilidad léxica. Salamanca. Disponible en www.dispolex.com.

Clark, H.h. 1970. "Word associations and linguistic theory". New horizons in Linguistics. Ed. J. LYONS. Londres: Penguin. 271-286.

Damasio, A. R. 1990. "Category related recognition defects as a clue to the neural substrates of knowledge”. Trends in Neurosciences, 13, 3: 95-98. Diccionario de La Rae. Vigésima segunda edición. 20 diciembre 2012 http://lema. rae.es/drae/. Diccionario Panhispánico de dudas. 2005. Madrid: Santillana.

Duque Pérez, Ma J. 1996. Los anglicismos en la informática. Tesis de licenciatura. Universidad de Las Palmas de Gran Canaria.

Durán Martínez, R. 2002. La lengua inglesa en la publicidad española: una aproximación pragmática. Tesis Doctorales: Universidad de Salamanca.

Fernández Calvo, R. 1994. Glosario básico inglés-español para usuarios de Internet. 05 Julio 2011.

Gerritsen, M., Nickerson, C., Van hooft, A., Van meurs, F. nederstigt, U., Starren, M., Crijns, R. 2007. English in product advertisements in Belgium, France, Germany, the Netherlands and Spain”. World Englishes, 26, 3: 291-315.

737 Vol. 3, núm. 4, octubre, 2017, pp. 720-738

Mayra A. Tejena-Macías 
Gómez Moreno, J. 1996. “¿Dumping o competencia desleal? ¿Servicio de catering o de abastecimiento? La invasión anglosajona en el lenguaje de la economía y la empresa”. Lenguas para fines específicos (V).

Eds S. Barrueco, E. Hernández, E. y L. Sierra. Alcalá de Henares: Universidad de Alcalá de Henares. $135-142$.

González Martínez, a., y orellana ramirez, p. 2006. “Anglicismos en el léxico disponible de la provincia de Cádiz (España)”. Boletín de Lingüística: Universidad Central de Venezuela: 3-21.

Hernández-Muñoz, n. 2006. Hacia una teoría cognitiva integrada de la disponibilidad léxica: el léxico disponible de los estudiantes castellano-manchegos. Tesis doctoral, Universidad de Salamanca.

Hernández-Muñoz, N., Izura, C., y Ellis, A. 2006. "Cognitive aspects of lexical availability." The European Journal of Cognitive Psychology 18, 5: 730-755.

Jiménez, J. y mandado, e. 2008. "Los anglicismos en la jerga electrónica”. Ponencia presentada en Congreso TAEE. Universidad de Vigo-Universidad de País Vasco.

López Chávez, J. 1992. “Alcances panhispánicos del léxico disponible”. Lingüística 4: 26-124. 